\title{
Jean Stengers
}

\section{Le rôle de l'opinion publique dans la genèse d'une guerre: 1870 et 1914}

Des dirigeants, en 1870 et en 1914, ont-ils subi l'influence, et l'influence éventuellement irrésistible, de l'opinion publique? Est-ce l'opinion publique qui a rendu la guerre inévitable? La question se pose essentiellement pour la France de 1870 et pour la Russie de 1914. Si l'on écoute les dirigeants eux-mêmes de ces deux pays, l'analogie entre les deux situations, de 1870 et de 1914, au premier abord, paraît frappante.

Dans une conversation avec un interlocuteur anglais, le 22 juillet 1870, Napoléon III déclare: J'aurais aimé pouvoir régler l'affaire de la candidature Hohenzollern de manière pacifique, but France has slipped out of my hand. I cannot rule unless I lead. This is the most national war that in my time France has undertaken, and I have no choice but to advance at the head of a public opinion which I can neither stem nor check.* ${ }^{1}$ Lorsque l'Empereur vient s'incliner devant son vainqueur, le 2 septembre, à Sedan, il répète: Je n'ai pas voulu cette guerre, «mais l'opinion publique m'y a forcé ${ }^{2}$.

Le Tsar Nicolas II, le 29 juillet 1914, télégraphie à Guillaume II: «An ignoble war

${ }^{1}$ Lettre signée *An Englishman publ. dans le Daily Telegraph du 25 juillet 1870. L'auteur, qui a été reçu aux Tuileries, reproduit des paroles que l'Empereur l'a autorisé, dit-il, à faire connaître sau peuple anglais». L'intérêt de cette déclaration, très proche des faits, a été relevé par Charles de Rémusat, Mémoires de ma vie, éd. p. Charles H. Pouthas, t. 5 (Paris 1967) 277-279, et par Michael Foot, The Origins of the Franco-Prussian War, in: New Cambridge Modern History, t. 10 (Cambridge 1964) 598.

${ }^{2}$ Jean Stengers, Aux origines de la guerre de 1870: gouvernement et opinion publique: in: Revue Belge de Philologie et d'Histoire 34 (1950) 701; abrégé ci-après en: Stengers, Guerre de 1870. L'Empereur fait la même déclaration à Bismarck (ibid. 702). Dans une brochure qu'il écrit durant sa captivité et qui'il fait paraître sous le nom du marquis de Gricourt, il dit de même: «Le pays a voulu la lutte ... L'Empereur (y a été) vivement encouragé si ce n'est contraint par les manifestations de l'esprit public, (Marquis de Gricourt, Des relations de la France avec l'Allemagne sous Napoléon III (Bruxelles s.d. - 1871) 49-50; sur la rédaction de cette brochure, cf. Comte $C$. de Monts, La captivité de Napoléon III en Allemagne, trad. franç. (Paris 1910) 175, Henri Welschinger, La Guerre de 1870. Causes et responsabilités, t.I (Paris ${ }^{4}$ 1911) 54-55, et la correspondance Ollivier-Napoléon III dans Emile Ollivier, Philosophie d'une guerre: 1870 (Paris 1910) 339-340). L'Impératrice Eugénie, jusqu'à la fin de sa vie, continuera à défendre elle aussi la même idée: Stengers, Guerre de 1870, 704 . 
has been declared to a weak country (c'est la Serbie). The indignation in Russia, shared fully by me, is enormous. I foresee that very soon I shall be overwhelmed by the pressure brought upon me, and forced to take extreme measures which will lead to war.* ${ }^{3}$ Ce télégramme se croise avec un message de Guillaume II au Tsar, expédié dans la soirée du 28 juillet: .I fully understand how difficult it is for you and your Government to face the drift of your public opinion." "Telle est bien la spressure à laquelle le Tsar est soumis, et à laquelle il craint de ne pas pouvoir résister.

Les ministres, un Ollivier ou un Gramont en 1870, un Sazonow en 1914, soulignent aussi cette pression qu'ils subissent, et font ressortir ce que sont les exigences de l'opinion. Emile Ollivier, dès le 5 juillet 1870, lorsqu'il explique à Lord Lyons, l'ambassadeur de Grande-Bretagne, qu'il est impossible de permettre à un Hohenzollern de monter sur le trône d'Espagne, lui dit: «Public opinion in France would never tolerate it. Any cabinet, any government which acquiesced in it would be at once overthrown. ${ }^{5}$ Au même Lord Lyons, le 12 juillet, et cela après l'annonce de l'abandon de la candidature Hohenzollern, le duc de Gramont explique que «public opinion was so much excited in France, qu'elle exigeait some more complete satisfaction from Prussia" 6.

Le 30 juillet 1914, Sazonow déclare à l'ambassadeur de Grande-Bretagne, Sir George Buchanan, parlant de la Russie: «Excitement in country has reached such a pitch that she cannot hold back if Austria refuses to make concession" (c'est le style du télégramme de Buchanan). Et Sazonow de souligner encore que si la Russie demeurait passive, "there would be a revolution in the country ${ }^{7}$.

${ }^{3}$ Outbreak of the World War. German Documents collected by Karl Kautsky, éd. p. Max Montgelas et Waltber Scbücking, trad. anglaise (New York 1924) 295; How the War began in 1914, being the diary of the Russian Foreign Office, éd. p. W. Cyprian Bridge (Londres 1925) 46. Guillaume II commente le télégramme du Tsar devant le Kronrat, en disant: :Sein Volk sei ausser sich, und selbst wenn er den Krieg mit Deutschland nicht wollte, so würde er von der öffentlichen Meinung overwhelmed werden. (relation de Tirpitz, dans Immanuel Geiss, Julikrise und Kriegsausbruch 1914, t. II (Hannover 1964) 274).

${ }^{4}$ Outbreak of the World War, 296-297; How the War began, 45-46.

${ }^{3}$ Lyons à Granville, 7 juillet 1870, Public Record Office, F.O. 27/1805; d'après le Blue Book dans Briefe, Aktenstücke und Regesten zur Geschichte der Hohenzollernschen Thronkandidatur in Spanien, éd. p. Richard Fester, t. II (Berlin 1913) 4.

${ }^{6}$ Lyons à Granville, 12 juillet 1870, Public Record Office, F.O. 146/1474 (minute) et 27/1806 (original); d'après le Blue Book dans le recueil de Fester, II, 109-110.

${ }^{7}$ Buchanan à Grey, 30 juillet 1914, dans British Documents on the Origins of the War 18981914, éd. p. G.P. Goocb et Harold Temperley, t. XI (Londres 1926) 191-192. Dans un article sur la crise de juillet 1914, j'ai commis l'erreur de considérer que la première phrase reflétait les vues, non de Sazonow, mais de Buchanan lui-même (Jean Stengers, July 1914, some reflections, in: Annuaire de l'Institut de Philologie et d'Histoire Orientales et Slaves 17 (1963-1965) 118; abrégé ci-après en: Stengers, July 1914). L'examen attentif du texte, de même que le recours au Confidential Print du Foreign Office, Further Correspondence respecting the War, July and August 1914 (Public Record Office, F.O. 438/2 et 371/2159), ne laissent pas de doute: c'est une déclaration de Sazonow. 
Mais tout ceci ne consiste-t-il pas à invoquer - ce qui est un cas fréquent dans les relations internationales - l'*alibi' de l'opinion publique, afin d'expliquer que l'on n'a pas de liberté d'action? Apparemment non. Apparemment, un Gramont, un Sazonow croient ce qu'ils disent. Gramont se sent poussé dans les reins par l’opinion. Lorsqu'il télégraphie, puis écrit à Benedetti, le 10 juillet 1870: "Vous ne pouvez vous imaginer à quel point l'opinion publique est exaltée. Elle nous déborde de tous côtés." "L'opinion publique s'enflamme et va nous devancer ${ }^{8}$, lorsqu'il lui télégraphie à nouveau le 12 juillet: «L'animation des esprits est telle que nous ne savons pas si nous pourrons la dominer ${ }^{9}$, rien ne permet de penser qu'il se sert là d'un *alibi»; il exprime des craintes réelles.

Ces textes, pris ici à titre de simples exemples, invitent donc à une comparaison du cas de la France de 1870 avec celui de la Russie de 1914.

Dans l'étude de ce problème, nous nous trouvons, notons-le immédiatement, devant deux traditions historiographiques complètement différentes.

Le rôle de l'opinion publique dans la genèse de la guerre de 1870 a été un thème de discussion dès l'époque même de la guerre, et la question n'a pas cessé depuis d'occuper les historiens, et souvent de les diviser. Les déclarations de Napoléon III à Sedan, le 2 septembre, avaient été immédiatement connues. Dans une circulaire du 17 septembre adressée aux représentants de la France à l'étranger, le nouveau ministre des Affaires étrangères, Jules Favre, réplique énergiquement: «Vainement ceux qui ont déchaîné sur la France ce redoutable fléau (c'est-à-dire la guerre) essayent-ils aujourd'hui d'échapper à la responsabilité qui les écrase en alléguant faussement qu'ils ont cédé au vœu du pays. Cette calomnie peut faire illusion à l'étranger ...; mais il n'est personne chez nous qui ne la repousse hautement comme une œuvre de révoltante mauvaise foi.. ${ }^{10}$ Et Michelet d'emprunter pour sa part le mode lyrique lorsqu'il évoque l'Empereur disant .La guerre, ce n'est pas moi, c'est la France qui l'a voulue : «Mensonge indigne et bas, le plus faux qui se fit jamais ... La scène est solennelle ici et mémorable. Elle rappelle celle de la Genèse où ce lâche Adam qui a peur, dit en tremblant: ‘Moi? Seigneur! ... C'est elle, c'est cette femmelà!, . 11

Dans la littérature historique sur 1870 , le problème est toujours resté présent. Jules Favre et Michelet ont trouvé des émules parmi les historiens républicains. Le 8 , le 9 , le 10 juillet 1870 , écrit Jean Jaurès, sla France presque tout entière voulait la paix, et elle aurait soutenu énergiquement le ministre, qui, d'une parole mesurée et ferme, aurait dénoncé les agités ${ }^{12}$. D’autres historiens, par contre, sans suivre com-

${ }^{8}$ Les Origines diplomatiques de la Guerre de 1870-1871, t. 28 (Paris 1931) 190 et 193; abrégé ci-après en: Origines diplomatiques.

9 Origines diplomatiques, 28, 255. Même télégramme à La Valette, à Londres: ibid. 28, 257-258.

${ }^{10}$ Journal officiel de la République française, 18 septembre 1870.

11 Jules Micbelet, La France devant l'Europe (Florence 2 février 1871) 64-65; rééd. (Genève 1946) 87.

12 Jean Jaurès, La Guerre franco-allemande 1870-1871, rééd. (Paris 1971) 233. 
plètement Napoléon III, font de l'excitation de l'opinion publique française un facteur relativement important. Des études spéciales - dont les conclusions ne concordent pas toujours - ont été consacrées au sujet par Malcolm Carroll, par Lynn M. Case, par le signataire de ces lignes ${ }^{13}$.

Pour 1914, au contraire, le problème de l'opinion, ou des opinions publiques, a été presque complètement absent des grandes controverses sur les origines de la guerre. Le livre, pourtant excellent, de $J$. F. Scott, Five weeks. The surge of public opinion on the eve of the Great War, publié en 1927, n'a eu pratiquement aucun écho - c'est un des grands méconnus de la littérature historique ${ }^{14}$. Le maître incontesté, en France, des études sur les origines de la guerre, Pierre Renouvin, écrivait en 1955 à propos de 1914: «Les mouvements de l'esprit public, pour autant que la recherche historique peut permettre d'apprécier leur rôle, ne semblent, nulle part, avoir exercé une impulsion ... Les mouvements d'opinion ... n'ont pas poussé les hommes d'Etat à l'action de force. ${ }^{15}$ Le maître américain, Bernadotte Schmitt, se prononçait en 1961 dans le même sens ${ }^{16}$. Nous avons essayé pour notre part - en sens inverse -, dans un article publié en 1966, d'attirer l'attention sur le cas de la Russie, et sur le poids en 1914 de l'opinion publique russe, ce qui est le thème que nous reprenons dans le présent exposé ${ }^{17}$.

Quelles sont, s'agissant à la fois de 1870 et de 1914, les questions à se poser? On peut, nous semble-t-il, en distinguer essentiellement quatre:

1. Quelles ont été, si l'on se livre à une analyse aussi objective que possible, les réactions de l'opinion?

2. Au moment de prendre ses grandes décisions, le pouvoir a-t-il agi avec le sentiment qu'il lui était impossible de résister à la contrainte de l'opinion?

3. L'opinion, en tout état de cause, rendait-elle ces grandes décisions inévitables?

4. Sans le facteur de l'opinion, la guerre aurait-elle pu être évitée?

${ }^{13}$ E. Malcolm Carroll, French public opinion on war with Prussia in 1870, in: American Historical Review 31 (1925-1926) 679-700; du même, French public opinion and Foreign affairs 1870-1914 (Londres 1931) 25 et sv.; Lynn M. Case, French opinion on war and diplomacy during the Second Empire (Philadelphie 1954) 241 et sv.; Stengers, Guerre de 1870. Voir aussi Aimé Dupuy, 1870-1871. La Guerre, la Commune et la presse (Paris 1959) et Rudolf von Albertini, Frankreichs Stellungnahme zur deutschen Einigung während des Zweiten Kaiserreichs, in: Schweizerische Zeitschrift für Geschichte 5 (1955).

14. It is the contention of the writer, écrivait Scott, athat the influence of public opinion in certain countries during the diplomatic crisis of the summer of 1914 was the most important factor in precipitating the war: (Scott, 19).

15 Pierre Renouvin, Le XIXe siècle. II. De 1871 à 1914, in: Histoire des relations internationales publ. sous la dir. de Pierre Renouvin (Paris 1955) 372. Voir dans le même sens son article, Les origines de la guerre de 1914, in: Le Monde, 29 juillet 1964.

${ }^{16}$ Bernadotte E. Schmitt, The relation of public opinion and foreign affairs before and during the First World War, in: Studies in diplomatic history and historiography in honour of G.P. Gooch, éd. p. A. O. Sarkissian (Londres 1961) 328.

17 Stengers, July 1914. 
Cette décomposition du problème peut paraître, bien entendu, quelque peu artificielle, mais de tels éclairages successifs offrent, nous semble-t-il, un bon cadre à la discussion.

Première question: Les réactions de l'opinion, analysées aussi objectivement que possible.

\section{A. $1870^{18}$}

La candidature Hohenzollern a suscité une réaction très forte, très vive, de l'opinion française: une véritable poussée de fièvre. La réaction a été pratiquement immédiate. Impossible, considère-t-on, de ne pas répondre à une telle provocation. L'ardeur quasi-belliqueuse qui sera celle, le 6 juillet, de la déclaration du duc de Gramont, va, dans les jours qui suivent, donner le ton. «Nous sommes ici en pleine ébullition ", écrit le 7 juillet le correspondant parisien d'un joumal belge. all serait inutile de dissimuler la vivacité de l'agitation qui s'est emparée de l'opinion publique, et dont les journaux, à quelque nuance qu'ils appartiennent, se font les interprètes. ${ }^{19}$ Un diplomate allemand à Paris note le 9 juillet que sles esprits sont montés ici contre la Prusse jusqu'au paroxysme, ${ }^{20}$.

La colère est d'autant plus vive que la candidature Hohenzollern apparaît comme la goutte d'eau qui fait déborder le vase. On a le sentiment d'avoir été, depuis 1866, depuis Sadowa, humilié par la Prusse ${ }^{21}$. «Depuis Sadowa», note - en raillant d'ailleurs - un journaliste, *il est admis que nous avons à l'orgueil national une plaie béante et saignante.."22 L'opposition, dans ses attaques contre le régime, s'est d'ailleurs plu à insister sur ce thème, à souligner l'humiliation de la France. "N'est-ce pas vous», lance Emile Ollivier, ‘qui depuis 1866 n'avez cessé de représenter l'œuvre de Sadowa comme une déchéance intolérable? ${ }^{23} \mathrm{La}$ formule de Thiers est qu'il faut sréparer Sadowa ${ }^{24}$. On avait à ce point remué le fer dans la plaie «qu'à la fin", observe un contemporain, «Sadowa semblait être une défaite française plutôt qu'une défaite autrichienne* ${ }^{25}$. Après cette longue humiliation, le défi

\footnotetext{
${ }^{18}$ Nous renvoyons pour les développements du sujet à notre exposé détaillé dans Stengers, Guerre de 1870.

${ }^{19}$ Echo du Parlement belge, 9 juillet 1870 .

${ }^{20}$ Cité par Eberbard Kolb, Der Kriegsausbruch 1870. Politische Entscheidungsprozesse und Verantwortlichkeiten in der Julikrise 1870 (Göttingen 1970) 100; abrégé ci-après en: Kolb, Kriegsausbruch.

${ }^{21}$ Analyse de ce sentiment dans Stengers, Guerre de 1870, 710-712.

${ }^{22}$ Le Figaro, 12 juillet 1870.

${ }^{23}$ Discours du 15 juillet 1870 au Corps législatif, d'après le Journal officiel de l'Empire français, $1870, \mathrm{n}^{0} 193$.

${ }^{24}$ Il la répète encore dans son discours du 15 juillet 1870 au Corps législatif: Adolphe Thiers, Discours parlementaires, éd. p. M. Calmon, t. 12 (Paris 1882) 653.

${ }^{25}$ Fernand Giraudeau, La vérité sur la campagne de 1870 (Marseille 1871) 103.
} 
de la candidature Hohenzollern: la coupe est pleine. Emile Ollivier lui-même dit à l'ambassadeur d'Autriche-Hongrie, avec une sorte de fureur concentrée: ‘Nous en avons assez des humiliations que la Prusse veut nous imposer.. ${ }^{26}$

Les témoignages des observateurs étrangers et ceux des observateurs français concordent pour faire ressortir la montée de la fièvre, la montée de l'excitation. Citons-les pour le 11 juillet: «Opinion surexcitée, (Beyens, ministre de Belgique à Paris) ${ }^{27}$ - The nation is extremely impatient, and as time goes on the war party becomes more exacting» (Lord Lyons) ${ }^{28}$ - «L'émotion ici est sérieuse et terrible ... Paris est dans une extrême agitation* (Ludovic Halévy) ${ }^{29}$ - «Une vive émotion règne dans Paris. La guerre prochaine est l'unique sujet de toutes les conversations. L'élan se prononce de plus en plus, les opinions s'effacent ou plutôt s'unissent dans un sentiment commun, on entend répéter partout: Il faut en finir!» (Correspondance du Journal de Bruxelles) ${ }^{30}$ :

Même après l'abandon de la candidature Hohenzollern, comme le gouvernement lui-même a annoncé que l'affaire n'est pas close, l'agitation des esprits, entretenue par une grande partie de la presse, persiste. Le 13 et le 14 juillet éclatent à Paris les premières manifestations de rue importantes. L'alarme des diplomates étrangers qui suivent la situation à Paris ne s'atténue pas, bien au contraire. Metternich, l'ambassadeur d'Autriche-Hongrie, télégraphie à Vienne le 14 juillet, à $15 \mathrm{~h}$. 20 (c'est avant qu'il n'apprenne le contenu de la «dépêche d'Ems»): „Opinion publique commence à peser sur le gouvernement au point que la paix paraît désormais impossible.* ${ }^{31}$

C'est sur ce fond d'agitation et même, chez beaucoup, d'une surexcitation qui n'a pas cessé, que le gouvernement devra délibérer des suites à donner à la dépêche d'Ems.

Dans cette opinion, dont nous tentons d'esquisser ici la physionomie, le sentiment dominant, qui deviendra très vite un sentiment passionnel, est que l'on ne peut tolérer l'attitude de la Prusse, qu'il faut à tout prix - et le prix sera, s'il le faut, la guerre - que la Prusse recule. Mais il existe incontestablement une frange, très

${ }^{26}$ Hermann Oncken, Die Rheinpolitik Kaiser Napoleons III. von 1863 bis 1870 und der Ursprung des Krieges von 1870/71, t. 3 (Berlin 1926) 402; abrégé ci-après en: Oncken, Rheinpolitik.

${ }^{27}$ Beyens à d'Anethan, 11 juillet 1870, Bruxelles, Arch. du Ministère des Affaires étrangères, Correspondance politique, Légations, France.

${ }^{28}$ Lyons à Granville, 11 juillet 1870; Public Record Office, F.O. 27/1805. Dépêche publiée dans le Blue Book avec la date erronée du 12 juillet, ce qui a créé une certaine confusion chez les historiens: voir Stengers, Guerre de 1870, 717 n.4.

${ }^{29}$ Lettre à Prévost-Paradol du 11 juillet, dans Ludovic Halévy, Carnets, éd. p. Daniel Halévy, t. II (Paris 1935) 169.

${ }^{30}$ Journal de Bruxelles, 12 juillet 1870.

31 Oncken, Rheinpolitik, t. 3, 437. Lord Lyons télégraphie lui aussi à peu près à la même heure: The excitement, especially in the army, is so great that it is doubtful whether the Emperor will or can with safety withstand the cry for wars (F.O. 27/1806; non publié dans le Blue Book). 
minoritaire sans doute, mais extrêmement bruyante, qui accueille avec une ardeur presque joyeuse l'idée que la France, grâce à l'incident Hohenzollern, puisse enfin infliger à la Prusse la leçon qu'elle mérite. Elle souhaite visiblement la guerre. C'est l'idée de la abonne guerre - une guerre évidemment victorieuse, personne n'en doute - qui restaurera le prestige de la France, et consolidera par le fait même le régime impérial. On trouve cette idée chez des hommes politiques, chez des journalistes de droite. C'est aussi celle, apparemment, de l'Impératrice Eugénie ${ }^{32}$.

Certains, à l'époque, ont été à ce point impressionnés par ces voix qui criaient à la guerre qu'ils ont pu croire qu'il s'agissait de la voix du pays. L'envoyé personnel du Roi d'Italie à Paris, le comte Vimercati, va dans ce sens lorsqu'il télégraphie le 14 juillet à Victor-Emmanuel II: ‘Opinion publique veut la guerre. ${ }^{33}$ Mais c'est surtout - et ceci est capital - Napoléon III qui se laisse aller à cette grossière erreur d'interprétation. L'Empereur a manifestement cru, en 1870, que la France, que ale pays, voulait la guerre. Ce qu'il écrit et dit lui-même est, à cet égard, parfaitement clair. A la nouvelle de la renonciation du prince de Hohenzollern, il déclare: „C’est la paix!», mais il ajoute: «Le pays sera désappointé. ${ }^{34}$.Je sais bien •, dit-il à Nigra, le ministre d'Italie, sque l'opinion publique, excitée comme elle est, eût préféré une autre solution, la guerre .... ${ }^{35}$.

L'erreur, chez Napoléon III, provient sans doute de plusieurs causes. De l'influence de son entourage, presque certainement. Mais aussi peut-être de la projection inconsciente, sur ce qu'il regarde comme «le pays*, de sa propre psychologie. L'Empereur, en juillet 1870 , est un homme vacillant - malade, ce qui explique sans doute beaucoup de choses, mais surtout vacillant. Il passe par des sentiments divers

${ }^{32}$ Il y a sur Eugénie toute une littérature, qu'il serait trop long d'analyser ici. Voir une page qui nous paraît bonne dans Adrien Dansette, Du 2 Décembre au 4 Septembre. Le Second Empire (Paris 1972) 388. Il faut traiter avec une certaine méfiance, notons-le, ce qu'a écrit sur le sujet la veuve d'Emile Ollivier (Marie-Thérèse Ollivier, L'Epouse de l'Empereur. Souvenirs personnels, in: Revue de Genève, février 1921; de la même, J'ai vécu l'agonie du Second Empire, éd. p. Anne Troisier de Diaz, Paris 1970). Nous attribuons par contre une grande valeur au témoignage d'une demoiselle d'honneur de l'Impératrice, Marie de Larminat. Celle-ci écrit de Saint-Cloud, vraisemblablement le 13 juillet 1870 . *Tout le monde ici, l'Impératrice en tête, désire tellement la guerre qu'il me paraît impossible que nous ne l'ayons pas (Comtesse des Garets, Souvenirs d'une demoiselle d'honneur, Auprès de l'Impératrice Eugénie, éd. p. Marie-Louyse des Garets (Paris 1928) 187).

${ }^{33}$ Documenti Diplomatici Italiani, 1re série, 1861-1870, vol. 13 (Rome 1963) 77; abrégé ciaprès en: DDI.

${ }^{34}$ Lettre à Emile Ollivier du 12 juillet, dans Origines diplomatiques t. 28, 251-252.

${ }^{35}$ Costantino Nigra, Ricordi diplomatici 1870, in: Nuova Antologia 140 (1895) 10. L'Empereur, écrit Emile Ollivier, était sinquiet à cause de la déception qu'allait éprouver le pays de ne pas vider définitivement sa querelle avec la Prusse (Emile Ollivier, L'Empire libéral, t. 14 (Paris 1909) 239; du même, Philosophie d'une guerre: 1870 (Paris 1910) 169). Ollivier cite aussi ces paroles révélatrices de Napoléon III dans la soirée du 14 juillet: •Voyez dans quelle situation un gouvernement peut se trouver parfois; n'aurions-nous aucun motif avouable de guerre, nous serions cependant obligés de nous y résoudre pour obéir à la volonté du pays!s (L'Empire libéral, t. 14, 373; Philosophie d'une guerre, 270). 
et contradictoires. Lorsqu'il tente des démarches secrètes pour obtenir le désistement de Léopold de Hohenzollern, c'est évidemment dans l'espoir de sauver la paix $^{36}$. Mais à d'autres moments, non moins évidemment, le désir qui le saisit, lui aussi, est celui d'une «bonne guerre». Le 11 juillet, dans la soirée, il s'entretient avec Metternich du meilleur moyen de "rendre la guerre inévitable. "Vous voyez,, mande l'ambassadeur à son gouvernement, "que l'on désire marcher (le con*, bien entendu, désigne avant tout l'Empereur), pensant avec raison, je crois, ne jamais trouver une meilleure occasion. ${ }^{37}$. Le 12 juillet, après le désistement, recevant $\mathrm{Ni}$ gra et Vimercati, Napoléon III ne leur fait pas mystère de ses réactions: il n'est pas satisfait «car il aurait préféré la guerre ${ }^{38}$. L'Empereur, observe Vimercati, était *véritablement désolé ${ }^{39}$. De là à projeter ses sentiments sur "le pays*, psychologiquement, il n'y a qu'un pas. Ce sera là, chez l'Empereur, un redoutable égarement.

\section{B. $1914^{40}$}

Soulignons-le d'entrée de jeu: dans la Russie de 1914, l'excitation a été grande, mais on ne trouve nulle part le souhait d'une «bonne guerre». Chez aucun dirigeant, pas plus que dans aucune fraction de l'opinion, on ne perçoit le sentiment que la guerre pourrait être profitable à la Russie, qu'elle pourrait être pour elle une solution heureuse. Certains observateurs, devant la houle qu'ils voyaient monter, ont pu s'y tromper. Ainsi de Maurice Paléologue, l'ambassadeur de France à Saint-Pétersbourg, qui déclare le 31 juillet 1914 à un membre de son entourage: Sazonow travaille inlassablement à essayer de sauver la paix, mais «il sera l'homme le plus

${ }^{36}$ Ce sont les démarches par la filière Olozaga-Strat - bien connues depuis le récit d'Emile Ollivier -, et celles par l'intermédiaire du Roi des Belges. *A la demande pressante mais très secrète de l'Empereur Napoléon, mandait Léopold II à la Reine Victoria, ejai écrit au Prince Léopold de Hohenzollern pour lui dire que de sa sagesse, de son désistement au trône d'Espagne, dépendait le repos du monde. (The Letters of Queen Victoria, 2e série, t. II, éd. p. Geonge Earle Buckle (Londres 1926) 25-26; la lettre du Roi des Belges à Léopold de Hohenzollem a été publiée par Michel Huisman, Juillet 1870. La candidature Hohenzollern et la famille royale de Belgique, in: Le Flambeau, janvier 1932).

${ }^{37}$ Oncken, Rheinpolitik, t. 3, 427. On trouve un écho de cette conversation entre l'Empereur et Metternich dans une dépêche de Beyens, qui était en contact étroit avec Metternich. •On a délibéré, écrit Beyens le 12 juillet, ssur les prétextes à prendre dans le cas où le dernier grief (= la candidature Hohenzollern) ferait défaut ... Hier encore l'Empereur a repris l'examen de ce points (Beyens à d'Anethan, 12 juillet 1870; fonds cité).

${ }^{38}$ Nigra à Visconti Venosta, 13 juillet 1870, dans DDI, 1re série, vol.13, 70.

${ }^{39}$ Vimercati à Victor-Emmanuel II, 12 juillet 1870, dans DDI, ibid., 60. Nigra, dans une note rédigée postérieurement, a contesté la présence de Vimercati à une entrevue avec l'Empereur le 12 juillet (voir ibid., 60 n. 1); sa mémoire le trahissait car Beyens, dans une dépêche du 13 juillet, parle d'une réception à la fois de Nigra et de Vimercati (Beyens à d'Anethan, 13 juillet 1870; fonds cité).

${ }^{40}$ Pour une analyse plus détaillée que celle que nous présentons ici, voir Stengers, July 1914. 
vilipendé, le plus impopulaire de son pays s'il évite la guerre ${ }^{41}$. C'était croire que l'opinion russe voulait la guerre. La méprise était grossière. Peut-être, comme dans le cas de Napoléon III en 1870, s'expliquait-elle en partie par la projection que $\mathrm{Pa}$ léologue faisait de sa psychologie personnelle: celle d'un patriote français qui, du fond du cœur, ne pouvait s'empêcher de songer à la srevanche. ...42

L'arrière-plan psychologique sur lequel se profile le conflit qui va éclater n'est pas du tout, dans la Russie de 1914, celui que l'on avait trouvé dans la France de 1870. Ce n'est pas la rancœur et l'animosité à l'égard d'un rival dangereux. C'est un sentiment d'une toute autre nature: le sentiment de solidarité que la Russie, la grande nation slave, éprouve pour ses efrères», les Slaves des Balkans, dont elle se juge la protectrice naturelle. L'ambassadeur britannique à Saint-Pétersbourg, Sir George Buchanan, fait là-dessus, avant 1914, d'excellentes observations. *There is one question in Russia on which public opinion cannot be disregarded. In its Slav sympathies all Russia is united, écrit-il le 9 octobre $1912^{43}$. Et le 15 mai 1913: -Militant Panslavism may be limited to a small section of Russian public opinion, but I do believe that every true Russian has at heart a vague sentiment of national solidarity and any war which closely affected Slav interests would at any rate be gladly endured if not welcomed. ${ }^{44}$

En 1914, l'homme qui sentit sans doute le mieux ce qu'allait être la signification de ce facteur psychologique profond, fut Jean Jaurès. Dans son discours de Vaise, le 25 juillet - le dernier discours qu'il ait prononcé en France - Jaurès s'écriait: “Citoyens, la note que l'Autriche a adressée à la Serbie est pleine de menaces, et si l'Autriche envahit le territoire slave, si les Germains, si la race germanique d'Autriche fait violence à ces Serbes qui sont une partie du monde slave et pour lesquels les Slaves de Russie éprouvent une sympathie profonde, il y a à craindre et à prévoir que la Russie entrera dans le conflit.. ${ }^{45}$

Analyse d'une admirable prescience. L'ultimatum autrichien à la Serbie va provoquer en Russie une indignation générale. all n'y a personne ici», écrit le 25 juillet le correspondant d'un journal belge à Saint-Pétersbourg, saussi bien dans ce qu'il est convenu d'appeler les hautes sphères, que dans les masses qui lisent les jour-

${ }^{41}$ Journal de la Marquise de Laguiche, femme de l'attaché militaire français à Saint-Pétersbourg; copie entre les mains de sa fille, la Princesse de Merode-Westerloo, à qui j’adresse ici mes vifs remerciements.

42 Un officier français qui rend visite à Paléologue le 31 juillet écrit: •M. Paléologue paraît tout à fait certain de la guerre et s'en réjouit presque, en songeant que la situation actuelle est la plus favorable que l'on ait jamais pu espérers (L'Esprit public en Russie. Journal d'un mobilisé, in: Le Correspondant, 10 septembre 1914, 756).

${ }^{43}$ British Documents on the Origins of the War, t. IX, 1re partie, 770.

44 Id., t. IX, 2e partie, 792-793. Sur ce sentiment de solidarité, voir Fernand Grenard, La Révolution russe (Paris 1933) 144-145, et Stengers, July 1914, 121-124.

${ }^{45}$ Alfred Rosmer, Le mouvement ouvrier pendant la guerre. De l'Union Sacrée à Zimmerwald (Paris 1936) 485; Jean Jaurès, CEuvres, éd. p. M. Bonnafous, t.9 (Paris 1939) 383. 
naux et se rendent plus ou moins compte de ce qui se passe, il n'y a personne, disje, qui ne considère l'acte de l'Autriche comme un défi, comme une offense sanglante faite à la Russie. ${ }^{46}$ Un Suédois établi dans la capitale russe notait le lendemain ce qu'il entendait dire "de toute part": «L'Autriche, ce pays haï et méprisé, osait tenir un tel langage et avoir de telles exigences envers une nation si proche du cœur de la Russie, placée sous sa protection, une nation de la même race et de la même foi ... Un coup en pleine face de toute la nation russe! ${ }^{47}$ Après l'attaque contre la Serbie, l'indignation va se faire explosive, violente. Les diplomates, les journalistes étrangers en sont les témoins. Ils parlent d'ailleurs moins d'indignation que d'،enthousiasme». «Enthousiasme», dans leurs rapports, est le mot-clé. «An extraordinary wave of enthusiasm is sweeping over the country , télégraphie le correspondant du Times le 29 juillet $^{48}$. Cet enthousiasme, c'est celui des manifestants qui emplissent de leurs cris les rues de Saint-Pétersbourg, de Moscou, d'Odessa, des Russes de toutes classes, des ouvriers mêmes - qui, à Saint-Pétersbourg passent d'un jour à l'autre d'une grève révolutionnaire à des démonstrations patriotiques ${ }^{49}$-, d'une Russie qui clame qu'elle veut courir au secours de ses frères serbes. Même les petites villes de province sont gagnées par la fièvre, signale le 31 juillet le correspondant du Matin. C'est, écrit-il, sl'immense et magnifique élan de la race slave, qui vient se précipiter au secours de ses frères victimes de la plus odieuse, de la plus inqualifiable agression ${ }^{50}$. Le ministre de Bulgarie à Saint-Pétersbourg, le général Dimitrieff, parle successivement, dans ses télégrammes du 30 et du 31 juillet, d'un enthousiasme grandissant pour la guerre avec l'Autriche", et d'un mouvement populaire en faveur de la guerre qui prend des eproportions incroyables ${ }^{51}$.

Le diagnostic le plus impressionnant est sans doute celui, le 30 juillet, du correspondant de la Frankfurter Zeitung. La guerre, télégraphie-t-il de Saint-Pétersbourg, stient à un fil. Des nouvelles alarmantes paraissent dans la presse russe. Et d'ajouter: "Natürlich steigern solche Alarmnachrichten die an sich hochgespannte Stimmung, die bereits das Mass überschritten hat, innerhalb dessen man annehmen durfte, die leitenden Staatsmänner würden sich ihr entziehen können, ${ }^{52}$ - la tension a atteint un tel degré que les dirigeants ne sont plus en mesure d'y résister ${ }^{53}$.

${ }^{46}$ Etoile belge, 2 août 1914.

47 Svenska Dagbladet, 31 juillet 1914; cf. Stengers, July 1914, 125-126.

48 The Times, 31 juillet 1914, p. 8.

49 Stengers, July 1914, 132-133; Alexander Kerensky, The crucifixion of liberty (Londres 1934) 175; Bertram D. Wolfe, An Ideology in power. Reflections on the Russian Revolution (New York 1969) 93.

${ }^{30}$ Le Matin, ler août 1914.

${ }^{51}$ Die Bulgarischen Dokumente zum Kriegsausbruch 1914, in: Die Kriegsschuldfrage 6 (1928) 249 et 251 .

${ }^{32}$ Frankfurter Zeitung, 31 juillet 1914.

${ }^{53}$ Mon analyse, dans July 1914, était fondée notamment sur le matériel original relatif à la Russie recueilli dans les principaux organes de presse de plusieurs pays européens - les jour- 
Ces deux brèves descriptions, de 1870 et de 1914, doivent être l'une et l'autre accompagnées d'un tempérament considérable. Nous avons, à propos de la fièvre de 1914, parlé avant tout de Saint-Pétersbourg, ou encore de Moscou ou d'Odessa. En 1870 , c'est avant tout Paris qui est en scène, et l'on pourrait sans doute y ajouter Marseille et quelques autres grandes villes ${ }^{54}$. Mais que dire de la campagne, des paysans français? Que dire, en 1914, en Russie, des immenses masses paysannes? Georges Sand, dans une lettre du 14 juillet 1870, marque bien le contraste entre Paris et son village de Nohant. "Plauchut, note-t-elle, "m'écrit que Paris est rugissant d'enthousiasme. Ce n'est pas la même chose en province. On est consterné.. ${ }^{35}$ Un contemporain relève de même dans son journal, à propos de l'agitation de $\mathrm{Pa}$ ris: «Les lettres que les députés recevaient de leurs électeurs attestaient une effervescence analogue dans les grands centres, et un calme véritable dans les campagnes.* ${ }^{56}$ En Russie, en 1914, reconnaît de son côté le baron de Taube, si "Pétersbourg, Moscou et les grandes villes témoignaient ... de leur ferme volonté de relever, comme il convenait à un grand peuple, le gant jeté à la Russie", il «était difficile de pénétrer dans le fond des sentiments du véritable peuple russe, de l'immense

naux britanniques, français, allemands, autrichiens, belges, hollandais, suisses, suédois et roumains - ainsi que dans des journaux américains. J'y ajoute maintenant la presse italienne, dont les correspondants en Russie fournissent des informations et des commentaires de la même intensité: La Stampa, de Turin, du 31 juillet 1914 (télégramme de Saint-Pétersbourg du 30 juillet parlant de la afervore di guerran), le Corriere della Sera, de Milan du 1er août 1914 (télégramme de Saint-Pétersbourg du 31 juillet), La Tribuna, de Rome, du 2 août 1914 (avec une importante correspondance de Saint-Pétersbourg, de juillet, signée ‘Lwows), et surtout le Giornale d'Italia du 3 août, contenant une longue correspondance de Saint-Pétersbourg d'Armando Zanetti, datée du 28 juillet. Zanetti écrit: .Il pubblico di Pietroburgo, Mosca e di qualche altra grande città, imbevuto di idee panslavistiche, educato, dalla coscenza più o meno diritta dei popoli occidentali, ha un concetto di dignità nazionale e di necessità politiche di una grande potenza, sente vivamente la necessità per la Russia in questo momento ... di riaffermarsi grande potenza, di giungere fino alla guerra pur di evitare una irreparabile annichilazione del proprio prestigio.. L'analyse de Zanetti, dont nous ne citons que ce court passage, est la plus poussée que nous ayons trouvée dans la presse occidentale. Armando Zanetti, jeune journaliste en 1914 - il était arrivé à Saint-Pétersbourg en 1913, à lầge de vingt-trois ans - a encore lu et commenté cinquante ans plus tard mon étude sur July 1914; il est décédé en 1977.

34 Voir notamment une allusion à Marseille dans une lettre de Napoléon III à La Valette du 23 juillet 1870. L'Empereur écrit: sL'enthousiasme qui existe en France est quelque chose d'incroyable. Le préfet de Marseille écrivait quelques jours avant que la guerre ne fût déclarée: Une paix, même bonorable, ne satisferait pas l'opinion publique, et si Marseille, ville pacifique par excellence, est sur ce diapason, figurez-vous le reste» (Adrien Dansette, Du 2 Décembre au 4 Septembre, 387-388).

35 Georges Sand, Correspondance, t. 6 (Paris 1884) 3.

${ }^{36}$ Emest Pinard, Mon Journal, t. II (Paris 1892) 42-43. 
population rurale, ${ }^{57}$. Sans doute, dans sa très grande majorité, avant que n'arrive l'ordre de mobilisation générale, ignorait-elle même ce qui se passait ${ }^{58}$.

L'oopinion publique* dont nous faisons si gxand cas n'était donc, tant en 1870 qu'en 1914, qu'une opinion restreinte. Le terme même d'aopinion publique*, dans ces conditions, n'est-il pas abusif? Notons que c'est celui que les contemporains, pratiquement sans exception, employaient constamment. Surtout, ce qui justifie l'usage du terme est que cette opinion, même restreinte, était celle qui, politiquement, comptait. C'est elle, essentiellement, qui pèse sur le régime, c'est d'elle, essentiellement, que le régime dépend. Le gouvernement impérial, s'il voulait éviter des troubles, devait regarder vers Paris, et non vers Nohant. Et personne, en Russie, en 1914, ne pouvait douter - ne fût-ce simplement qu'en observant les grèves de Saint-Pétersbourg, et les barricades que dressaient les grévistes - de l'endroit où, pour le gouvernement du Tsar, se situerait éventuellement le péril. L'،opinion publique, était là oú étaient aussi les poudrières.

Deuxième question: Le pouvoir, au moment de prendre ses décisions cruciales, a-t-il eu le sentiment qu'il lui était impossible de résister à la contrainte de l'opinion?

Dans ces relations pouvoir-opinion, une question préalable se pose: le pouvoir, en fait, ne subit-il pas la pression d'une opinion qu'il a lui-même contribué à former, à exciter.

Si l'on prend le cas de 1870, il est évident que la déclaration Gramont du 6 juillet, par sa forme même - des paroles qui sonnent la fanfare, et qui ont surpris et choqué des diplomates étrangers ${ }^{59}$ - a donné un coup de fouet à l'opinion ${ }^{60}$. Mais, il est facile de le voir, elle n'a fait qu'accentuer une réaction qui était déjà très vive avant même que Gramont ne monte à la tribune, et qui, en tout état de cause, aurait certainement eu une force considérable.

En 1914, à Saint-Pétersbourg, les manifestations de rue ont sans doute bénéficié

${ }^{57}$ Baron M. de Taube, La politique russe d'avant-guerre et la fin de l'Empire des tsars, 1904-1917. Mémoires (Paris 1928) 380-381.

${ }^{58}$ De bonnes pages sur le monde paysan dans Bertram D. Wolfe, An Ideology in power, 94-96. Une remarque frappante du général Broussilov, dans ses Mémoires: .Even after war was declared, the drafts arriving from the interior of Russia had not the slightest notion what the War had to do with them ... The men were led like sheep to the slaughter without knowing why. (General A. A. Brussilov, A soldier's note-book 1914-1918 (Londres 1930) 37).

59 Voir Kolb, Kriegsausbruch, 100-105. Relevons à ce propos la réaction très caractéristique de Jules Devaux, le chef de cabinet de Léopold II, qui écrit à Beyens, le 8 juillet, dans une lettre privée (une lettre où le «nous» représente évidemment avant tout le Roi des Belges): «Nous concevons ici la colère de l'Empereur Napoléon et nous lui donnons raison. Mais nous donnons tort à son gouvernement d'avoir, au Corps législatif, engagé l'amour-propre de la France, au point où ils l'ont fait, sur des semi-informations» (Bruxelles, Arch. du Ministère des Affaires étrangères, Papiers Beyens).

${ }^{60}$ Elle a, écrivait Buloz, spassionné, l'opinion (Chronique de la quinzaine, in: Revue des Deux Mondes, 15 juillet 1870, 508). Beyens emploie le même verbe dans sa dépêche du 6 juillet (dans sa correspondance diplomatique, fonds cité). 
à certains moments de la collaboration de la police ${ }^{61}$. Mais ce n'est là qu'un élément tout à fait mineur, et on peut dire marginal.

Il est donc impossible de soutenir que le pouvoir a subi le contrecoup de ce qu'il avait lui-même engendré. Pour l'essentiel, l'opinion avec laquelle il a dû compter s'était formée en dehors de lui.

A-t-il eu le sentiment qu'il lui était impossible d'y résister? Passons en revue à ce sujet les décisions majeures de 1870 et de 1914 .

\section{A. 1870}

Deux moments majeurs: la demande de garanties du 12 juillet, et, les 14-15 juillet, la décision de faire la guerre.

La demande de garanties a été élaborée et décidée, dans l'après-midi du 12 juillet, dans un tête-à-tête entre l'Empereur et son ministre des Affaires étrangères ${ }^{62}$. $\mathrm{Ni}$ Napoléon III ni Gramont n'ont jamais révélé le secret de cette délibération lourde de conséquences. Gramont dira le lendemain à Metternich que al'opinion très montée en France réclame cette solution* ${ }^{63}$. Quand on connaît d'autres déclarations de Gramont, et sa correspondance avec Benedetti - nous avons cité ces textes au début de notre article ${ }^{64}$-, quand on connaît les fantasmes que l'Empereur se faisait au sujet du epays», on incline à penser que Napoléon III et son ministre se sont effectivement imaginé qu'ils devaient agir comme ils le faisaient pour satisfaire aux exigences de l'opinion. L'explication est d'une très grande vraisemblance.

Mais d'autres ministres, une fois mis au courant de la demande de garanties, n'ont pas eu, apparemment, la même analyse. Même s'il subsiste beaucoup d'incertitude au sujet de ce qui s'est passé au Conseil des Ministres du 13 juillet $^{65}$, un fait est clair: le Conseil, le 13 juillet, a amorcé, dans la discussion avec la Prusse, une marche arrière. La majorité des ministres ne se sentaient donc manifestement pas tenus à une attitude intransigeante.

A ce moment, tout était encore possible. Comment les dirigeants français, devant une réponse prussienne autre que la gifle de la dépêche d'Ems, auraient-ils réagi? On ne peut se livrer à ce sujet qu'au jeu des hypothèses. Comme Bismarck 61 Stengers, July 1914, 126-127.

${ }^{62}$ Steefel a bien montré que la scène a dû se passer aux Tuileries, et non à Saint-Cloud comme on l'avait toujours dit. Ceci exclut donc la présence de l'Impératrice (Lawrence $D$. Steefel, Bismarck, the Hohenzollem candidacy and the origins of the Franco-German war of 1870 (Cambridge, Mass. 1962) 155-156).

${ }^{63}$ Oncken, Rheinpolitik, t. 3, 432.

64 Voir aussi Stengers, Guerre de 1870, 724-725, ainsi que les paroles de Gramont et d'Ollivier à Werther lorsqu'ils réclament - peu avant que ne soit élaborée la demande de garanties une lettre du roi de Prusse: «Les deux Ministres (faisaient) ressortir qu'ils avaient besoin d'un arrangement de ce genre pour calmer l'émotion des esprits. (Origines diplomatiques, 28, 462).

${ }^{65}$ Remarques très judicieuses à ce sujet dans Kolb, Kriegsausbruch, 131-132. 
l'écrivait, d'une plume perçante, dans une lettre du 10 juillet: „Man könne politische Entschliessungen zorniger Leute beiderlei Geschlechts eben so wenig vorhersagen wie das Wetter, ${ }^{66}$. Nous sommes ici en face d'un de ces «si» de l'histoire qui sont pratiquement insolubles.

Mais le fait net, incontournable, a été la publication et la communication par la Prusse à des gouvernements étrangers de la dépêche d'Ems. C'est là-dessus - en même temps d'ailleurs que sur des nouvelles relatives aux préparatifs militaires prussiens qui faisaient croire que la Prusse était décidée à la guerre ${ }^{67}-$ que le gouvernement français a délibéré le 14 et le 15 juillet, et c'est là-dessus qưil a décidé lui-même la guerre.

S'agissant de l'opinion publique et de l'influence qu'elle a pu exercer, réglons d'abord un point essentiel. Une très vieille version des faits - que l'on trouve d'ailleurs encore chez des auteurs récents ${ }^{68}$ - affirme que la publication de la dépêche d'Ems aurait provoqué à Paris, dans la foule parisienne, une violente explosion de colère qui n'aurait pas manqué évidemment d'impressionner le gouvernement. Cela est faux. L'annonce de la dépêche d'Ems n'a pas eu, dans l'opinion française, un effet immédiat, ni même un effet rapide, car la nouvelle a été noyée dans un flot d'informations diverses et contradictoires, où le public avait du mal à se retrouver. Cela n'a donc pas été un coup de tonnerre. Nous croyons en avoir autrefois apporté la démonstration: au moment où l'Empereur et ses ministres délibèrent, le texte en main, la réaction de l'opinion ne s'est pas encore déclenchée ${ }^{69}$.

Dans ce grand débat des 14-15 juillet, en dehors des soucis d'ordre militaire, deux considérations majeures, manifestement, ont été présentes à l'esprit de ceux qui devaient décider de la guerre ou de la paix:

- les exigences de l'honneur national: la France avait été souffletée

- l'anticipation de ce que serait la réaction du pays à cet outrage lorsqu'il le connaîtrait.

D'après les témoignages - et spécialement celui d'Emile Ollivier - ce second facteur a sans aucun doute pesé sur la décision (avec la formule: «On jetterait de la

${ }^{66}$ Fritz Stern, Gold und Eisen. Bismarck und sein Bankier Bleichröder (Francfort 1980) 170-171 (lettre dictée à sa femme).

${ }^{67}$ Elément bien mis en valeur dans l'analyse de Langer, Red Rag and Gallic Bull. The French decision for War 1870, in: William L Langer, Explorations in Crisis. Papers on International History (Cambridge, Mass. 1969) 157-159 et 164. .Dans la journée du 14», déclarera Gramont, nous reçumes de différents côtés des informations qui s'accordaient pour montrer la mobilisation des troupes prussiennes s'effectuant avec rapidité. (Enquête parlementaire sur les actes du gouvernement de la Défense Nationale. Dépositions des témoins, t. I. (Versailles 1872) 107).

${ }^{68}$ Voir notamment Aimé Dupuy, 1870-1871. La Guerre, la Commune et la presse (Paris 1959) 12; Steefel, Bismarck, the Hohenzollern candidacy, 208-209; Otto Pflanze, Bismarck and the development of Germany. The period of unification 1815-1871 (Princeton 1963) 456; Jasper Ridley, Napoleon III and Eugénie (New York 1979) 561.

${ }^{69}$ Voir sur tout ceci Stengers, Guerre de 1870, 35-44. Résumé dans Langer, Red Rag and Gallic Bull, 149. 
boue sur nos voitures et on nous huerait, si nous ne répondions pas à l'outrage comme il se doit) ${ }^{70}$, mais c'est l'honneur et la dignité de la France, de toute évidence, qui ont été au cœur du débat. Comment pouvait-on concevoir une nation fière qui ne relève pas l'affront public qu'elle avait subi: ce serait une France humiliée, abaissée. Dans les pages qu'Emile Ollivier a écrites sur ce thème, il y a sans doute une bonne dose de rhétorique (. Javais beau sophistiquer, argumenter, me débattre contre l'évidence, elle m'étreignait, me brisait, me subjuguait, et j'en revenais toujours à la même conclusion: la France vient d'être insultée volontairement, grossièrement, nous serions des gardiens infidèles de son honneur si nous le supportions* - «Après ce soufflet de Bismarck, la paix ne pouvait plus être que la paix dans la faiblesse, dans l'humiliation, dans l'abaissement» - «Il ne nous était plus permis de perdre notre temps en sentimentalités inutiles et périlleuses: nous n'avions qu'à accepter la rencontre à laquelle on nous obligeait. - «L'offense était directe, palpable, sanglante, voulue ${ }^{71}$ ). Mais ce serait certainement une erreur, parce que cette rhétorique est un peu ampoulée, d'en décrier la valeur historique: une certaine grandiloquence convient bien à l'expression de sentiments qui, à l'époque même, étant donné qu'il s'agissait de l'honneur national et de l'art français de la parole, devaient nécessairement se traduire par du pathos.

Ecoutons ce que Gramont déclarera à la Chambre, dans le débat du 15 juillet: -Messieurs, il suffit de ce fait que le gouvernement prussien a informé tous les cabinets de l'Europe qu'il avait refusé de recevoir notre ambassadeur et de continuer à discuter avec lui. Cela est un affront pour l'Empereur et pour la France. Et si, par impossible, il se trouvait dans mon pays une chambre pour le supporter ou pour le souffrir, je ne resterais pas cinq minutes ministre des Affaires étrangères. ${ }^{72}$ Gramont sera acclamé.

La manœuvre de Bismarck avait pleinement réussi.

${ }^{70}$ Marquis de Gricourt, Des relations de la France avec l'Allemagne, 46 (voir sur cette brochure ci-dessus n. 2); Ollivier, L'Empire libéral, t. 14, 373, et Philosophie d'une guerre, 270.

${ }^{71}$ Ollivier, Empire libéral, t. 14, 372, 380 et 555, et Philosophie d'une guerre, 269, 273 et 334. Dès le lendemain des événements, on le notera, Emile Ollivier insiste avec force sur ce thème dans sa correspondance: voir ses lettres à Napoléon III du 27 septembre 1870 (•Nous n'avons déclaré (la guerre) que forcés par le procédé du roi de Prusse, par la sréponse d’Ems» comme le dit avec orgueil Mommsen), à Ernest Ollivier du 24 décembre 1870 (*La Prusse a commis envers nous un de ces actes qu'aucune nation n'a jamais supportés sans signer sa déchéance morale et politiquen) et à Napoléon III encore du 28 décembre 1870 ( $S$ Supposez qu'un jour votre gouvernement ait fait afficher sur les murs de Paris (comme le roi Guillaume l'a fait à Ems et à Berlin) que les propositions anglaises sur la dénonciation du traité de commerce ayant déplu à Votre Majesté, elle refuse de recevoir de nouveau lord Lyons; non seulement Gladstone et Bright, mais les bonnets de coton anglais eux-mêmes eussent demandé la guerre. Si la France n'avait pas fait cette guerre, elle tombait dans la boues) - Emile Ollivier, Journal 1846-1869, t.II, éd. p. Theodore Zeldin et Anne Troisier de Diaz (Paris 1961) 448; du même, Lettres d'exil 1870-1914, in: Revue des Deux Mondes, 6e période, 51 (1919) 725; $d u$ même, Philosophie d'une guerre, 340 . 


\section{B. 1914}

Trois moments capitaux à distinguer: la décision du gouvernement, puis du Tsar, d'intervenir, par les armes s'il le faut, en faveur de la Serbie; la mobilisation partielle; enfin la mobilisation générale.

1. La décision de soutenir la Serbie, et de le faire par les armes s'il le fallait, a été prise par le Conseil des Ministres russe dès le 24 juillet 1914, c'est-à-dire l'aprèsmidi même du jour où avait été annoncé l'ultimatum autrichien.

Nous ignorions absolument tout, jusqu'il y a peu, du déroulement de ce Conseil - c'était la lacune la plus grave dans notre connaissance des événements de 1914 . Nous disposons aujourd'hui du témoignage d'un des participants, le ministre des Finances, Peter Bark. Il est capital ${ }^{73}$.

Citons, en suivant le récit de Bark, les interventions majeures dans la discussion gouvernementale.

Sazonow, le ministre des Affaires étrangères, qui prend la parole le premier, comme il se doit: The moment had come when Russia, faced with the annihilation of Serbia, would lose all her authority if she did not declare herself the defender of a Slavonic nation threatened by powerful neighbours ... Serbia could not accede to the clauses of the ultimatum without losing her entire independence. The Austrian army would then enter Serbia and that small country would suffer total defeat after a few battles. Russia could not remain a passive spectator whilst a Slavonic people was being deliberately trampled down ... Our policy had always been directed towards the defense of the Slavs. If Russia failed to fulfil her historic mission, she would be considered a decadent State and would henceforth have to take second place among the Powers... The information at his disposal had convinced him that Austro-Hungary and Germany were resolved to deal a decisive blow at Russian authority in the Balkans by annihilating Serbia. Under these circumstances, he thought that the only way in which Russia could ward off the blow and safeguard her authority in international affairs was to take up a firmer attitude than previously and to intervene in favour of Serbia. ${ }^{74}$

72 Journal officiel de l'Empire français, 1870, $\mathrm{n}^{\circ} 193$.

${ }^{73}$ Peter Bark, Memoirs, Ms.; New York, Columbia University Libraries, Rare Book and Manuscript Library, Bakhmeteff Archive, Bark Papers, Box 1. Il s'agit là de la traduction anglaise de mémoires que Bark avait rédigés en français (cf. dans les Bark Papers, Box 3, les lettres de Lady Bark à O. Locker-Lampson, du 24 février 1937, et à Mrs Macmillan, s.d.; voir aussi l'introduction de Gaston Riou au texte cité ci-après.) Un seul chapitre de l'original français a été publié: Peter Bark, Nicolas II, le dernier tsar (avec introduction de Gaston Riou) in: Hommes et Mondes, 8 (1953) 58-75. Sur Bark, qui, après avoir quitté la Russie en 1919, fit en Occident une seconde et brillante carrière - il est mort Sir Peter Bark -, voir Who was who 1919-1940, 64; Great Soviet Encyclopedia, t. 3 (Londres 1973) 23; l'obituary du Times, 18 janvier 1937; F. Sauvaire-Jourdan, Sir Peter Bark, in: Revue politique et parlementaire, 172 (1937) 438-448. Je remercie vivement M.J. L. De Paepe, Attaché scientifique à l'Académie Royale de Belgique, qui m'a assisté dans mes recherches sur Bark.

74 Memoirs, chap. 7, 7-13. 
Sazonow est soutenu par Krivoschéine, le ministre de l'Agriculture. Krivoschéine était, nous dit Bark, le «membre le plus influent du cabinet», et son intervention fit une impression profonde. Elle fut, dit-il, «the most instrumental in influencing our decisions ${ }^{75}$. Krivoschéine concluait lui aussi à la nécessité d'une attitude énergique, et il déclarait notamment, faisant allusion à ce que serait selon toute vraisemblance la réaction de l'opinion: aPublic and parliamentary opinion would fail to understand why, at this critical moment involving Russia's vital interests, the Imperial Government was reluctant to act boldly. ${ }^{76}$

Le Ministre de la Guerre et le Ministre de la Marine déclarèrent sobrement que hesitation was no longer in its place. ${ }^{77}$.

Bark lui-même se prononce en disant: *Since the honour, dignity and authority of Russia were at stake, the Finance Minister should adhere to the opinion of the majority of the Cabinet. ${ }^{78}$

Les autres ministres déclarèrent qu'ils partageaient les vues de Sazonow et de Krivoschéine.

Le Président du Conseil, Goremykine, pouvait donc constater l'unanimité du gouvernement. Il conclut aby saying that it was the Imperial Government's duty to decide definitely in favour of Serbia. This was the only course of action which could bring about a peaceful solution of the crisis caused by the Austrian ultimatum. However, if in spite of the efforts made by the Russian Government to prevent an armed conflict, war became inevitable, Russia should be ready to make the sacrifices required of her.» Il résumait la pensée unanime du gouvernement par la formule: « We do not want war, but we do not fear it." ${ }^{79}$

Le Conseil, on le voit, n'agit pas sous la pression de l'opinion, qui ne s'est pas encore manifestée - bien que l'on devine ce qu'elle pourra être - mais essentiellement en fonction de ce qu'exigent l'honneur, la dignité, la mission historique de la Russie, si celle-ci veut conserver son rang en Europe. Gardons ceci à l'esprit pour la suite de notre analyse.

L'attitude résolue - résolue jusqu'à la guerre, s'il le faut - du Conseil des Ministres du 24 juillet, se traduit, sur le plan militaire, par l'adoption du principe d'une mobilisation partielle, c'est-à-dire d'une mobilisation contre l'Autriche. Le Conseil charge ales ministres de la Guerre et de la Marine de solliciter l'assentiment de Sa Majesté Impériale pour la déclaration de mobilisation des quatre districts militaires

\footnotetext{
75 Ibid., 7 et 16 . Sur l'importante stature politique de Krivoschéine, voir D. C. B. Lieven, Russia and the origins of the First World War (Londres 1983) 142. On notera que Lieven est, à ma connaissance, le seul historien qui ait fait usage jusqu'ici des mémoires de Bark (voir 141-144).

${ }^{76}$ Memoirs chap. 7, 13-16.

${ }^{77}$ Ibid., 17.

78 Ibid., 17-19.

79 Ibid., 19-20.
} 
de Kiev, Odessa, Moscou et Kazan, ainsi que des flottes de la Baltique et de la Mer Noire, et ceci en fonction du développement ultérieur des événements ${ }^{80}$.

Le lendemain, 25 juillet, le Tsar approuve les résolutions du Conseil. Le Journal du Comité de l'Etat-Major général russe porte:

-Le chef de l'Etat-Major général a informé les membres du Comité que Sa Majesté l'Empereur avait bien voulu déclarer qu'il était nécessaire de soutenir la Serbie, même si l'on devait proclamer la mobilisation et entamer des opérations militaires, ce qui ne pouvait cependant pas se faire avant que les troupes autrichiennes n'aient franchi la frontière serbe ... S'il se trouvait nécessaire de proclamer la mobilisation, en raison du fait que les opérations doivent se limiter à l'Autriche seulement, Sa Majesté a ordonné que la mobilisation se fasse dans les districts militaires de Kiev, Odessa, Kazan et Moscou.» ${ }^{81}$

Les dés sont jetés. On peut dire que tout ce qui suivra ne sera plus que soubresauts et péripéties.

2. Le 29 juillet, dans la matinée, l'Autriche ayant déclaré la guerre à la Serbie, le Tsar signe l'ukaze de mobilisation partielle de l'armée russe, pour les quatre districts prévus ${ }^{82}$. C'est l'application en quelque sorte automatique de la décision de principe proposée par le gouvernement le 24 juillet et adoptée par le Tsar le 25.

3. La suite, qui est un peu chaotique, est l'histoire, avant tout, des vacillations du Tsar. Le 29, au début de la soirée, Nicolas II se laisse convaincre de remplacer l'ordre de mobilisation partielle par un ordre de mobilisation générale. Mais ensuite, à peine deux heures plus tard, il se ravise. Il recule devant la guerre. .The Emperor", écrit Bark, "was filled with an overwhelming desire to prevent at all costs a war whose cruelty appalled him. ${ }^{83}$ Vers $10 \mathrm{~h} .1 / 2-11 \mathrm{~h}$. du soir, le Tsar annule donc l'ordre de mobilisation générale, et l'on en revient à la mobilisation partielle, qui prendra cours le 29 à minuit. Dans la journée du 30, Sazonow autant que les militaires font pression sur le Tsar pour qu'il se résolve à la mobilisation générale. Vers 5 heures de l'après-midi, enfin, il en donne l'ordre - un ordre cette fois définitif, qui sera affiché sur les murs de Saint-Pétersbourg dans les petites heures du 31 juillet.

Dans cette grande agitation des 29-30 juillet, la nécessité de la pression militaire sur l'Autriche-Hongrie et, s'il le faut, d'une intervention armée pour soutenir la Serbie, ne sont, il faut le souligner, nullement remises en cause. Le maintien, en tout état de cause, de la mobilisation partielle, le prouve. Comme il s'agit là de ce

${ }^{\text {so }}$ Die Internationalen Beziehungen im Zeitalter des Imperialismus, trad. allemande éd. p. Otto Hoetzsch, 1re série, t. 5 (Berlin 1934) 25-26; Immanuel Geiss, Julikrise und Kriegsausbruch 1914, t.I (Hannover 1963) 354-355; du même, July 1914. The outbreak of the First World War: selected documents (Londres 1967) 186-187.

${ }^{81}$ Die Internationalen Beziehungen, ibid., 67; non repris dans le recueil de Geiss.

${ }^{82} \mathrm{Je}$ renvoie ici, et pour ce qui suit, à une étude à paraître prochainement dans un ouvrage collectif: Jean Stengers, 1914; the safety of cyphers and the outbreak of the First World War (dans Exeter Studies in History, vol. 14).

${ }^{83}$ Bark, Memoirs, chap. 7, 35. 
qu'exige, à grands cris, l'opinion publique, ce n'est pas de ce que réclame l'opinion que s'occupent, dans leurs discussions, le Tsar, ses ministres et les chefs militaires. Le Tsar redoute avant tout la guerre avec l'Allemagne, c'est-à-dire la guerre générale. Il cherche à l'éviter. Aujourd'hui, comme nous lisons dans les cartes de l'Allemagne, nous savons que les espoirs qu'il pouvait nourrir à cet égard étaient pure futilité. La mobilisation partielle de l'armée russe suffisait à rendre l'affrontement général inévitable: même si la Russie en était restée là, l'Allemagne lui aurait adressé un ultimatum et la séquence des événements aurait été la même que celle qui allait se dérouler un peu plus tard à la suite de la mobilisation générale ${ }^{84}$. Mais le Tsar croit que la mobilisation générale ouvre la porte sur l'abîme, et il hésite, il recule. Ses généraux, eux, lui remontrent qu'une mobilisation qui n'est que partielle risque de compromettre la mobilisation générale, et de compromettre par conséquent les chances militaires de la Russie. Ce débat est militaire, il est politique, mais, apparemment, la grande ombre de l'opinion publique ne plane pas sur lui.

On voit, en fin de compte, ce qu'est la profonde analogie entre les décisions gouvernementales françaises de 1870 et la prise de position du gouvernement russe en 1914. Les 14-15 juillet 1870, en France, le 24 juillet 1914, en Russie - car c'est ce jour-là que tout s'est joué - ce qui est en cause avant tout, ce ne sont ni des questions de sécurité, ni des questions d'intérêt, au sens classique du mot, mais l'honneur du pays et son rang en Europe. De part et d'autre, la préoccupation majeure est celle de ne pas déchoir. La France serait humiliée, la Russie tomberait au second rang, elle perdrait son autorité: ce que les dirigeants français et russes ne peuvent pas laisser rabaisser est la dignité et la grandeur de leur pays. Leur pays est provoqué, ils se doivent de relever le défi.

Troisième question: Une opinion publique qui rendait la guerre en tout état de cause inévitable?

Dans les trois grandes décisions de 1870 et de 1914 qui ont mené à la guerre et dans lesquelles le facteur de l'opinion publique a joué, nous en avons trouvé une (la demande de garanties) où ceux qui se sont imaginé agir sous la contrainte de l'opinion se sont laissé impressionner très largement par un fantasme, une seconde (la décision des 14-15 juillet 1870) où la considération de l'opinion publique, bien que présente et importante, a été éclipsée par d'autres, plus prégnantes, et une troisième enfin (la décision russe du 24 juillet 1914) où l'on a certes tenu compte de ce qu'allaient être, selon toute vraisemblance, les réactions de l'opinion russe, mais qui a été guidée elle aussi avant tout par des considérations différentes.

Mais il faut savoir s'élever au-dessus de l'événementiel: toutes modalités de décision mises à part, l'état de l'opinion en France et en Russie, après la dépêche d'Ems d'une part et après la déclaration de guerre à la Serbie d'autre part, ne devait-il pas,

84 Luigi Albertini, The origins of the War of 1914, trad. angl. éd. p. Isabella M. Massey, t. 2 (Londres 1953) 485 n. 1, et t.3 (Londres 1957) 30-31; L. C. F. Turner, Origins of the First World War (Londres 1970) 108. 
en tout état de cause, mener de manière inéluctable à la guerre? Dans les deux cas, pensons-nous, aucune hésitation n'est permise: la réponse est évidemment affirmative.

Le 14 juillet 1870 dans la soirée, l'ambassadeur britannique à Paris, Lord Lyons, écrivait à Granville: *After the article in the North German Gazette (c'est la publication de la dépêche d'Ems), war seems absolutely inevitable. ${ }^{85}$ Tout est dit là en une phrase. Il était inconcevable, une fois comprise la portée de la dépêche d'Ems - ce qui devait se produire tôt ou tard -, que le gouvernement pût résister à la fureur de l'opinion. Le pays serait entré en guerre avant le gouvernement.

En 1914, pouvait-on davantage concevoir que le gouvernement russe se borne à des protestations platoniques face à l'invasion de la Serbie? Souvenons-nous de ce que disait Sazonow: «There would be a revolution in the country.* Réfléchissant par la suite aux événements, un Kerenski, un Broussilov ne pensaient pas autrement. Kerenski disait: “Had the Russian Government yielded in 1914 and refused to intervene on the side of Serbia, public opinion in all circles would have been so irritated at the feebleness of this conduct, that the result might easily have been the Government's overthrow.* ${ }^{86}$ Broussilov écrivait de son côté: si le Tsar n'avait pas marché, «public resentment would have turned on him with such ferocity that he would have been tumbled from his throne, and the Revolution, with the support of the whole of the intelligentsia, would have taken place in 1914 instead of $1917{ }^{87}$.

Un régime aussi menacé que le régime tsariste - menacé, ne l'oublions pas, jusque dans sa propre capitale, par les grèves révolutionnaires de Saint-Pétersbourg n'aurait pu, sans danger mortel, aller à l'encontre du grand cri de ses propres partisans - du cri de guerre de ceux qui voulaient que la Russie relève le défi qui lui avait été lancé.

Quatrième question: S'il n'y avait pas eu l'opinion publique, y aurait-il eu malgré tout la guerre?

On trouvera peut-être à cette question une saveur de complication intellectuelle, si pas de jonglerie intellectuelle. Mais nous aimerions nous abriter derrière la grande ombre de Max Weber. Pour mesurer si un facteur causal jugé essentiel l'est véritablement, nous enseigne Max Weber, nous pouvons le supprimer "par la pensée, et "nous demander ensuite si, après cette sorte de modification des conditions du devenir, nous aurions pu nous attendre au même résultat, ou bien à un autre, et lequel, ${ }^{88}$.

${ }^{85}$ Public Record Office, Granville Papers, P.R.O. 30/29/85, et, d'après les Papiers Lyons, Richard Millman, British Foreign policy and the coming of the Franco-Prussian war (Oxford 1965) 193.

${ }^{86}$ Interview dans Jonatban French Scott, Five weeks. The surge of public opinion on the eve of the Great War (New York 1927) 177-178.

${ }_{87}$ Brussilov, A soldier's note-book, 40-41.

${ }^{88}$ Max Weber, Essais sur la théorie de la science, trad. franç. (Paris 1965) 300. Cf. Maurice Weyembergh, Le volontarisme rationnel de Max Weber (Bruxelles 1972) 262 et sv. 
Sans l'opinion, aurions-nous pu néanmoins, en 1870 et en 1914 , nous attendre à la guerre?

Dans une reconstruction logique du passé - car dans la méthode de Max Weber, tout tient évidemment à un raisonnement logique -, on peut, nous semble-t-il, répondre que oui.

Lorsque Lord Lyons, dans la soirée du 14 juillet 1870, sachant ce qu'est la dépêche d'Ems, écrit que la guerre «seems absolutely inevitable, il songe certainement en premier lieu aux réactions prévisibles de l'opinion, mais il songe aussi, on peut le penser, à tout le reste. Le reste, c'est l'impossibilité morale où se trouvera le gouvernement, s'il ne veut pas perdre la face, de ne pas répondre à l'outrage. Le reste, c'est aussi que l'outrage va atteindre des dirigeants déjà fort excités. Le duc de Gramont est excité, l'Empereur passe par des périodes d'excitation, Emile Ollivier est un caractère hautement émotif, impressionnable, qui a aussi ses moments d'exaltation guerrière. Le noble portrait en pied qu'Ollivier a tracé de lui-même dans L'Empire libéral, et dans lequel il garde toujours un admirable sang-froid et suit invariablement la ligne de conduite dictée par la raison, est parfaitement fallacieux. Les textes et les témoignages contemporains révèlent ses accès belliqueux, ou d'intransigeance exigeante ${ }^{89}$. Le 11 juillet, Metternich, qui a une information excellente, écrit qu'Ollivier cvoudrait d'autres questions (à joindre) à celle de Hohenzollern pour rendre la guerre inévitable, ${ }^{90}$. Le 12 juillet, dans un entretien avec Werther, l'envoyé prussien, en compagnie de Gramont, Ollivier formule à l'égard du roi de Prusse des desiderata - c'est la fameuse alettre d'excuses, - qui annoncent à beaucoup d'égards la demande de garanties ${ }^{91}$. C'était véritablement, et d'avance, un encouragement à ce que sera la demande de garanties ${ }^{92}$.

Ces deux éléments - le fait que les dirigeants français étaient acculés à réagir, et le fait qu'ils étaient d'humeur pour le moins très vive - suffisaient, même sans l'opinion, à former ensemble un mélange explosif qui eût rendu la guerre certaine.

Opinion ou pas, les dirigeants russes, en 1914, étaient acculés de la même ma-

${ }^{89}$ Cf. Douglas $W$. Houston, Emile Ollivier and the Hohenzollem candidacy, in: French Historical Studies 4 (1965) 125-149, qui a cependant le tort de se fonder exagérément sur les rapports, assez sujets à caution, de l'agent secret Klindworth.

90 Oncken, Rheinpolitik, t. 3, 417. Beyens écrit le même jour que -M. Ollivier s'est grisé de la guerre comme du plébiscite. .Son langage ne diffère guère de celui des jeunes attachés de son cabinet: Il faut en finir - Il faut que le roi voie ce que c'est qu'un ministre du peuple, etc.? (Beyens à d'Anethan, 11 juillet 1870 ; fonds cité).

91 C'est ce qui ressort du rapport de Werther, qu'Ollivier a assez maladroitement contesté: voir Origines diplomatiques, 28, 459-465, et Pierre Muret, Emile Ollivier et le duc de Gramont les 12 et 13 juillet 1870, in: Revue d'Histoire moderne et contemporaine 14 (1910) 178 et sv.

${ }^{92} \mathrm{M}$. Pierre Guiral est donc à mon sens beaucoup trop indulgent pour Ollivier lorsqu'il considère que, dans la demande de garanties, ssa responsabilité n'est pratiquement pas engagées et qu'il an'est pour rien dans la guerre de 1870. (Pierre Guiral, Emile Ollivier et la politique extérieure, in: Regards sur Emile Ollivier, éd. p. Anne Troisier de Diaz (Paris 1985) 203 et 205). 
nière: le souci du rang, de la dignité, de l'autorité de la Russie, à lui seul, leur imposait leur décision.

Opinion ou pas, on devait donc, dans les deux cas, déboucher sur la guerre.

Emile Ollivier a écrit là-dessus une page frappante. Aurions-nous pu, se demande-t-il, agir autrement que nous ne l'avons fait? „Depuis 1870», écrit-il, «je me suis souvent imaginé à la tribune le 15 juillet, conseillant la résignation à l'outrage, et je me suis demandé comment j'aurais pu engager une nation sensible à l'honneur, confiante en l'invincibilité de son armée, à dévorer un procédé sans précédents et si manifestement insultant, comment j'aurais répondu aux huées de l'Assemblée et au mépris de tous les hommes de cœur: je n'ai rien trouvé. Il n'était pas humainement possible, dans les circonstances au milieu desquelles nous délibérions, d'agir autrement que nous ne l'avons fait..93

Le Tsar aurait pu sans doute, s'il avait vécu, écrire la même chose à propos de 1914.

Dans les deux cas - et c'est sans doute là l'analogie la plus fondamentale entre 1870 et 1914 -, le pays qui s'est décidé à la guerre s'est senti tenu, obligé de répondre à une provocation. En 1870 , il y avait même eu deux provocations successives la candidature Hohenzollern, puis la dépêche d'Ems. En 1914, l'ultimatum à la Serbie, puis l'attaque de l'Autriche formeront un tout.

Entre la ou les provocations de 1870 et celle de 1914, il faut cependant souligner une différence essentielle: en 1870, l'auteur de la seconde et majeure provocation, c'est-à-dire de la dépêche d'Ems - nous laissons ici de côté la question controversée de la candidature Hohenzollem - l'avait conçue pour provoquer la guerre; en 1914, l'Autriche-Hongrie et l'Allemagne, tout en acceptant l'idée d'une guerre générale, nourrissaient l'espoir que la Russie ne réagirait pas par les armes ${ }^{94}$.

Dans ces conditions, il est intéressant de le noter, on aurait pu s'attendre à ce que les observateurs étrangers, à l'époque, condamnent beaucoup plus sévèrement les premiers provocateurs (nous employons le pluriel car on n'avait pas encore pleinement identifié à l'époque le rôle personnel de Bismarck) qu'ils ne condamneront les seconds. Or le paradoxe est à cet égard assez extraordinaire: c'est le contraire qui s'est produit.

En 1914, l'Autriche-Hongrie et l'Allemagne ont bien été considérées, en général, comme les grandes coupables. En 1870, par contre, ce n'est pas sur la Prusse, mais sur la France que le blâme s'est principalement abattu. Le Times, le 16 juillet, publiait un éditorial flamboyant: .The greatest national crime that we have had the pain of recording in these columns since the days of the First French Empire has been consummated. War is declared - an unjust, but premeditated war. That dire

93 Ollivier, Empire libéral, t. 14, 559, et Philosophie d'une guerre, 338-339.

${ }^{94}$ Ceci va à l'encontre, il est inutile de le souligner, des thèses de Fritz Fischer. Il serait trop long d'expliquer ici pourquoi je ne puis les accepter. Voir notamment, dans le sens que j'indique, Luigi Albertini, The origins of the War of 1914, t. 2, 159-161. 
calamity ... is, it is now too clear, the act of France - of one man in France. ${ }^{95}$ La presse anglaise, en général, flétrit la France ${ }^{96}$. Des journaux belges, comme L'Etoile belge, reproduisent le jugement du Times ${ }^{97}$. «Votre gouvernement n'a pas bien engagé son affaire*, écrit Emile de Laveleye à Dupont-White le 2 août. "L'opinion partout est contre lui.» ${ }^{98}$ Le comte de Flandre, le frère de Léopold II, écrit à la Reine Victoria: aJe crois que la France cherchait un prétexte pour attaquer l'Allemagne, et on a saisi le premier venu. ${ }^{99}$ Léopold II lui-même explique "cette affreuse guerre en assurant que ala France veut s'agrandir et dominer le monde ${ }^{100}$. Elle mérite d'être écrasée, écrit-il encore, «pour avoir sans motifs commencé une guerre de pure ambition. ${ }^{101}$.

Ces jugements défavorables, sévères, féroces même parfois, s'expliquent par une série de causes cumulées: par un fond de vieille défiance à l'égard des ambitions du régime impérial; par l'impression désastreuse qu'ont faite les manifestations verbales belliqueuses qui ont précédé la guerre - ce qui fait apparaitre la guerre comme le prolongement en quelque sorte de ces cris belliqueux; par l'attitude jugée inadmissible de la France lorsqu'après avoir obtenu pleine satisfaction dans la question de la candidature Hohenzollern, elle a néanmoins continué à poursuivre la Prusse de ses exigences.

Mais on sera surtout frappé par l'accusation de Léopold II: avoir commencé la guerre «sans motifs*. Ceci montre que le Roi des Belges n'avait pas du tout mesuré l'importance, pour le gouvernement français, de la dépêche d'Ems. Il est en cela très représentatif de ses contemporains: en dehors de la France, cette importance n'avait guère été perçue ${ }^{102}$. La lecture de la presse - de la presse anglaise, par exemple, ou de la presse belge, du Times ou de l'Indépendance belge - l'explique parfaite-

95 The Times, 16 juillet 1870, p. 8 .

${ }^{96}$ Patrick Bury, L'opinion britannique et les affaires françaises de 1870, in: Revue d'Histoire diplomatique 84 (1970) 340-344; Richard Millman, British Foreign policy, 195. Thomas Huxley écrit le 18 juillet 1870 à un ami allemand: ‘Public opinion in England ... is entirely against France, (Leonard Huxley, Life and Letters of Thomas Henry Huxley, t. I (New York 1901) 359).

${ }^{97}$ L'Etoile Belge, 18 juillet 1870.

${ }^{98}$ Bruxelles, Académie Royale de Belgique, Archives, Lettres d'Emile de Laveleye à DupontWhite. Dans un mémoire rédigé en novembre 1870, sur «L'Allemagne et la Belgique pendant et après la guerre de 1870, Emile Banning écrit: ‘Quand éclata la guerre actuelle, l'opinion belge, s'inspirant exclusivement des principes de la justice internationale, se prononça presque à l'unanimité en faveur de l'Allemagne, contre la France agressive et conquérante. Les victoires des armes allemandes furent à nos yeux les victoires du droit. (Bruxelles, Archives Générales du Royaume, Papiers Banning, $\mathrm{n}^{0} 80$; Banning a publié ce texte dans une brochure anonyme, sous le même titre (Bruxelles 1870); voir Jean Stengers, Textes inédits d'Emile Banning (Bruxelles 1955) 28-29).

${ }^{99}$ Lettre du 16 juillet 1870, dans Robert Demoulin, Documents inédits sur la crise internationale de 1870, in: Bulletin de la Commission Royale d'Histoire 122 (1957) 141-142.

100 The Letters of Queen Victoria, 2e série, t. II, 45 (lettre du 24 juillet 1870).

${ }_{101}$ Robert Demoulin, Documents inédits, 218 (lettre du 30 octobre 1870).

102 Bien remarqué par Kolb, Kriegsausbruch, 136-137. 
ment: alors que la demande de garanties y occupe une grande place, qu'elle est analysée et commentée, la dépêche d'Ems, elle, n'est pas du tout mise en valeur. Nulle part on ne la place en pleine lumière. La France insultée n'a pas réussi à faire comprendre à l'Europe qu'elle l'était, à convaincre l'Europe qu'elle avait été provoquée $^{103}$. C'est peut-être là que réside l'ineptie majeure du gouvernement impérial.

La manœuvre de Bismarck a donc été doublement triomphante: sa provocation a produit l'effet qu'il souhaitait - tant en Allemagne d'ailleurs qu'en France -, et, en dehors de la France et de l'Allemagne, il n'en a pas payé le prix.

${ }^{103}$ Même le Blue Book anglais, à cet égard, l’a desservie. Gramont avait donné à Lord Lyons, le 15 juillet, une explication claire: «Nor indeed, lui disait-il, thad the King really treated M. Benedetti with the rough discourtesy which had been boasted of by the Prussian Government. But that Government had now chosen to declare to Germany and to Europe that France had been affronted in the person of her Ambassador. It was this boast which was the gravamen of the offence. It constituted an insult which no nation of any spirit could brook» (dépêche de Lyons du 15 juillet 1870; F.O. 27/1806). Ceci ne sera pas publié dans le livre bleu. 